\title{
Project YES! Youth Engaging for Success: A randomized controlled trial testing a peer mentoring approach among HIV- positive adolescents and young adults in Ndola, Zambia
}

Julie Denison

Virginia M. Burke

Sam Miti

Bareng A.S. Nonyane

Christiana Frimpong

See next page for additional authors

Follow this and additional works at: https://knowledgecommons.popcouncil.org/departments_sbsr-hiv

Part of the Public Health Commons

How does access to this work benefit you? Let us know!

\section{Recommended Citation}

Denison, Julie, Virginia M. Burke, Sam Miti, Bareng A.S. Nonyane, Christiana Frimpong, Katherine G. Merrill, Elizabeth A. Abrams, and Jonathan Kaunda Mwansa. 2021. "Project YES! Youth Engaging for Success: A randomized controlled trial testing a peer mentoring approach among HIV-positive adolescents and young adults in Ndola, Zambia," Project SOAR Final Report. Washington, DC: USAID I Project SOAR. 


\section{Authors}

Julie Denison, Virginia M. Burke, Sam Miti, Bareng A.S. Nonyane, Christiana Frimpong, Katherine G. Merrill, Elizabeth A. Abrams, and Jonathan Kaunda Mwansa 


\section{Project YES! Youth Engaging for Success}

peer mentoring approach among HIV-positive adolescents and young adults in Ndola, Zambia

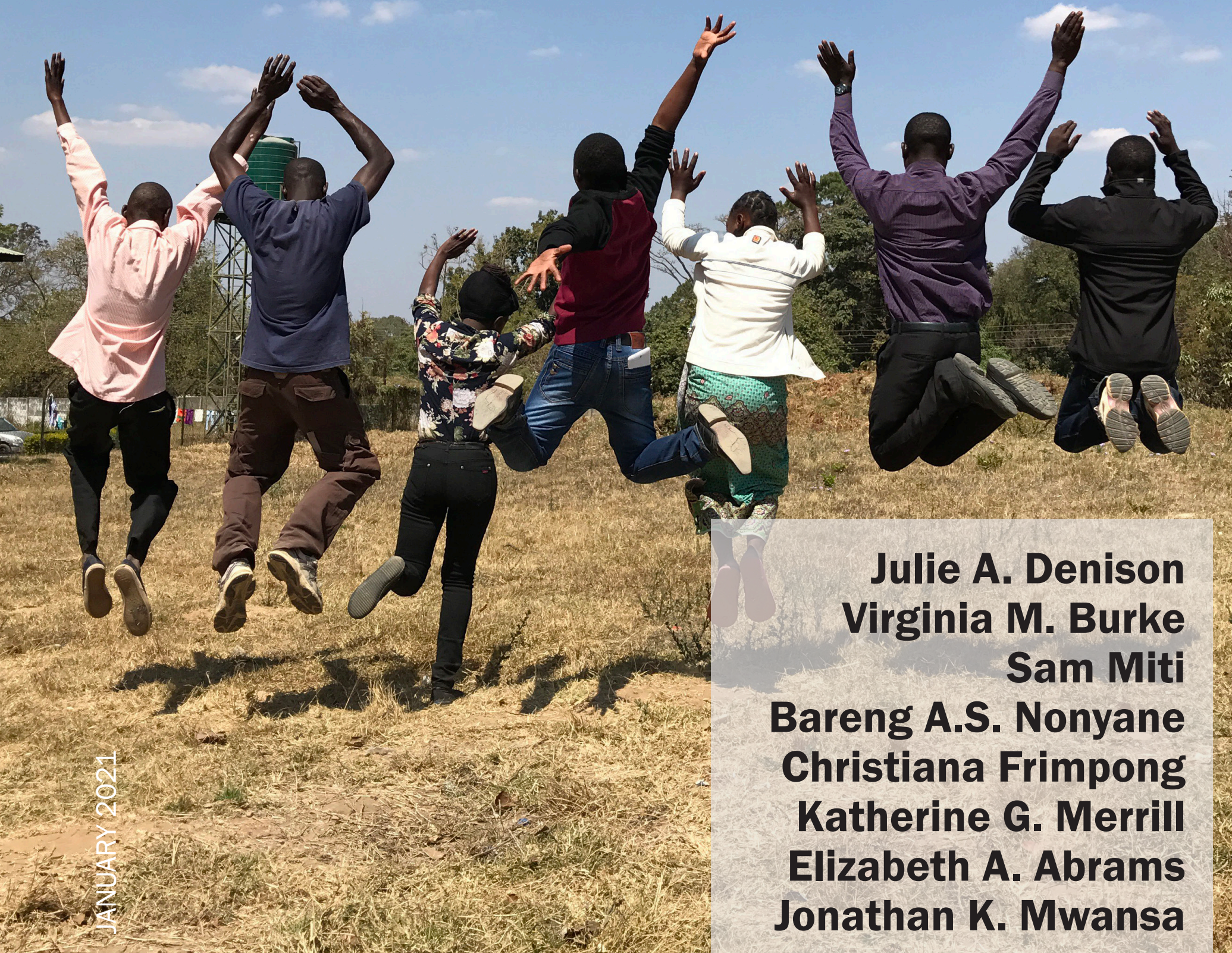

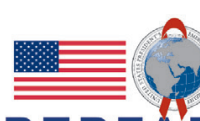

PEPFÂR
(1)

USAID
JOHNS HOPKINS

YSOAR 
Project SOAR

Population Council

4301 Connecticut Ave, NW, Suite 280

Washington, D.C. 20008 USA

Tel: +12022379400

Fax: +1202 2378410

projsoar.org
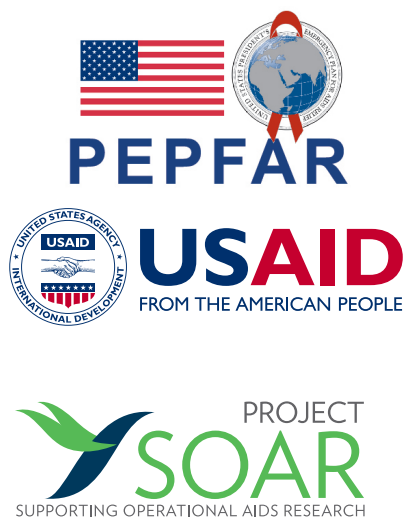

\section{- POPULATION COUNCIL}

Ideas. Evidence. Impact.
Project SOAR (Cooperative Agreement AID-OAA-A-14-00060) is made possible by the generous support of the American people through the United States President's Emergency Plan for AIDS Relief and the United States Agency for International Development (USAID). The contents of this report are the sole responsibility of Project SOAR and the Population Council and do not necessarily reflect the views of PEPFAR, USAID, or the United States Government.

Through operations research, Project SOAR will determine how best to address challenges and gaps that remain in the delivery of HIV and AIDS care and support, treatment, and prevention services. Project SOAR will produce a large, multifaceted body of high-quality evidence to guide the planning and implementation of HIV and AIDS programs and policies. Led by the Population Council, Project SOAR is implemented in collaboration with Avenir Health, Elizabeth Glaser Pediatric AIDS Foundation, Johns Hopkins University, Palladium, and The University of North Carolina.

The Population Council confronts critical health and development issues-from stopping the spread of HIV to improving reproductive health and ensuring that young people lead full and productive lives. Through biomedical, social science and public health research in about 50 countries, the Council works with our partners to deliver solutions that lead to more effective policies, programs, and technologies to improve lives worldwide. Established in 1952 and headquartered in New York, the Council is a nongovernmental, nonprofit organization with an international board of trustees.

Cover photo credit: @Katherine G. Merrill/Johns Hopkins University

Published in January 2021. (02021 The Population Council Inc.

Suggested citation: Denison, Julie A., Virginia M. Burke, Sam Miti, Bareng A.S. Nonyane, Christiana Frimpong, Katherine G. Merrill, Elizabeth A. Abrams, Jonathan K. Mwansa. 2021. "Project YES! Youth engaging for success: A randomized controlled trial testing a peer mentoring approach among HIV-positive adolescents and young adults in Ndola, Zambia," Project SOAR Final Report. Washington, DC: USAID | Project SOAR. 


\section{Project YES! Youth Engaging for Success: A randomized controlled trial testing a peer mentoring approach among HIV-positive adolescents and young adults in Ndola, Zambia}

Julie A. Denison ${ }^{1}$, Virginia M. Burke ${ }^{1}$, Sam Miti ${ }^{2}$, Bareng A.S. Nonyane ${ }^{1}$, Christiana Frimpong ${ }^{2}$, Katherine G. Merrill ${ }^{1}$, Elizabeth A. Abrams ${ }^{1}$, and Jonathan K. Mwansa ${ }^{2}$

1Johns Hopkins Bloomberg School of Public Health, Department of International Health, Baltimore, Maryland, USA

${ }^{2}$ Arthur Davison Children's Hospital, Ndola, Zambia

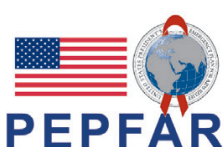




\section{ACKNOWLEDGMENTS}

This study represents the possibilities when young people are engaged in a supportive environment with adult guidance. The youth peer mentors did a tremendous job learning and implementing their role as peer mentors for young people living with HIV. The team also acknowledges with deep gratitude and thanks the adolescent and young adult clients who engaged in this study. Their stories and hope continue to inspire us. We also thank Ms. Teresa Peterson, the project's Training and Capacity Building Specialist, for her time and amazing ability to help young people transform into successful and professional peer mentors. The team also thanks the health care providers and study team members without whom this study could not have happened. We also wish to acknowledge the special role of the research advisory board, chaired by Dr. Sam Phiri, that helped keep the study accountable to the priorities and needs of youth in Zambia. 


\section{TABLE OF CONTENTS}

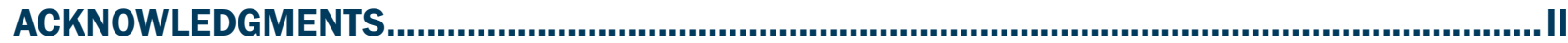

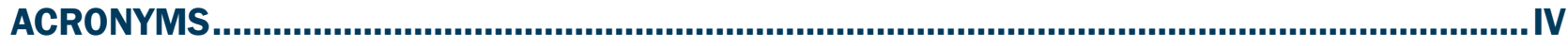

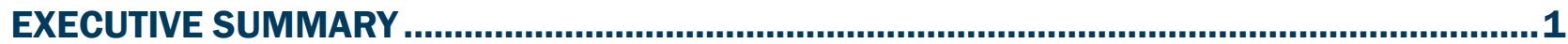

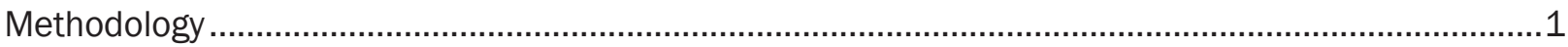

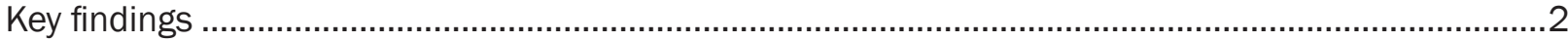

Conclusion and recommendations ........................................................................................

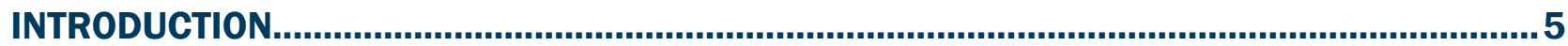

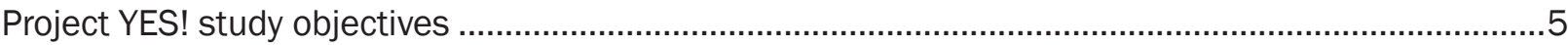

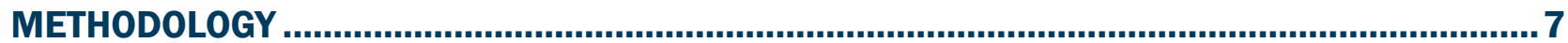

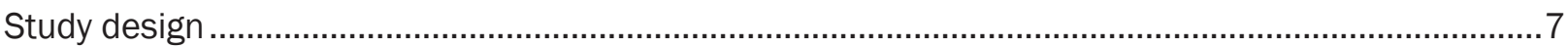

Description of intervention .........................................................................................................

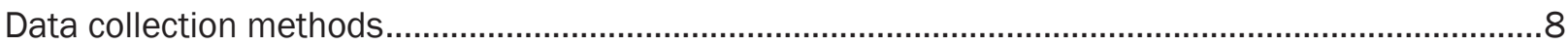

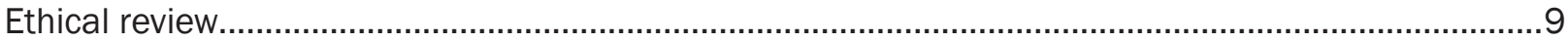

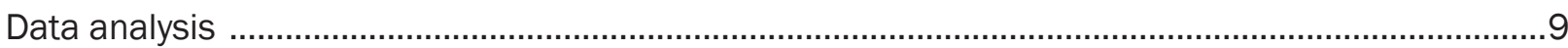

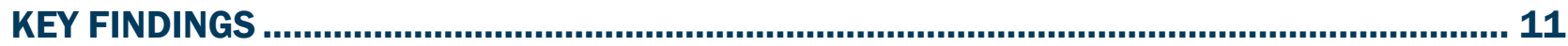

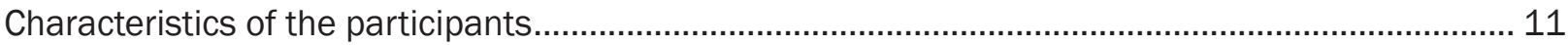

Objective 1 and 1A: Primary analysis on Project YES! impact and stratified analyses ..................... 12

Objective 2: Qualitative analyses of YPM, HCP, AYA, and caregiver IDIs ......................................... 15

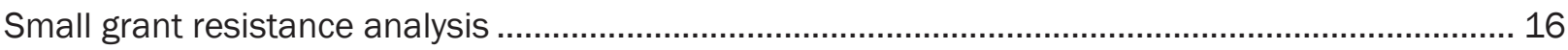

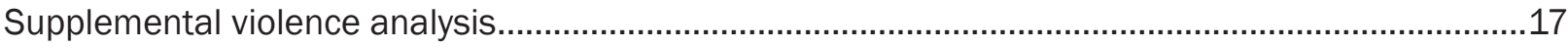

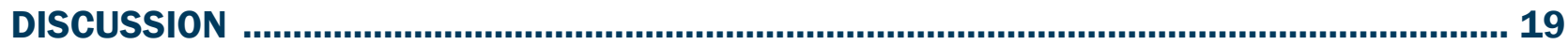

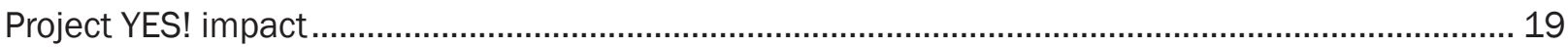

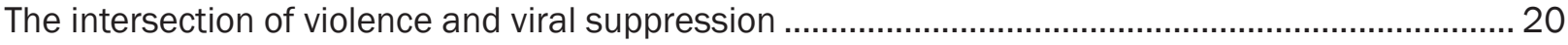

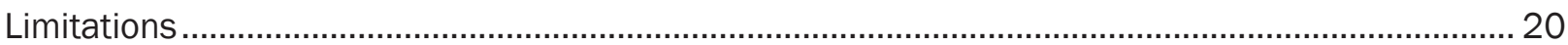

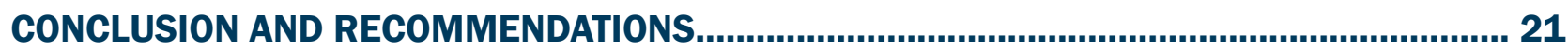

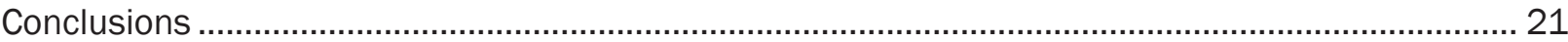

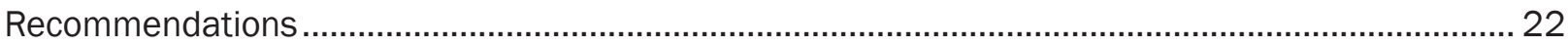

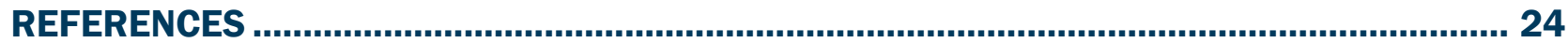




\section{ACRONYMS}

\begin{tabular}{|c|c|}
\hline ART & Antiretroviral treatment \\
\hline AYA & Adolescents and young adults \\
\hline GEE & Generalized estimating equation \\
\hline $\mathrm{HCP}$ & Health care provider \\
\hline HIV & Human immunodeficiency virus \\
\hline HIVDR & Human immunodeficiency virus drug resistance \\
\hline IDI & In-depth interview \\
\hline $\mathrm{MOH}$ & Ministry of Health \\
\hline Project YES! & Youth Engaging for Success \\
\hline RCT & Randomized controlled trial \\
\hline TAN & Technical Advisory Network \\
\hline VL & Viral load \\
\hline VS & Viral suppression \\
\hline WHO & World Health Organization \\
\hline YPM & Youth peer mentor \\
\hline
\end{tabular}




\section{EXECUTIVE SUMMARY}

Only about one-third of 15- to 24-year-olds living with HIV have achieved viral suppression in Zambia, a lower proportion than any other age group (Ministry of Health [MOH] 2019). Internalized stigma (self-stigma) including feelings of worthlessness, shame, and blame because one is living with HIV, had also been found to be common among HIV-positive adolescents in Ndola, Zambia (Denison et al. 2018). In addition, little is known about experiences of violence among adolescents and young adults (AYA), despite the fact that Zambia has one of the highest rates of partner violence in the world and violence has been associated with poor outcomes among adult women. This study examined the impact of Project YES!, a youth peer mentoring program, with well-trained youth employed at the clinic level, on improving viral suppression (VS) and reducing internalized stigma among HIV-positive AYA attending four HIV clinics in Ndola, Zambia.

\section{METHODOLOGY}

Two hundred seventy-six consecutively recruited AYA (15 to 24 years of age) were enrolled in a randomized controlled trial that was conducted in four HIV clinics in Ndola, Zambia (a children's hospital, an adult hospital, and two primary care facilities). Participants were randomly assigned to either an intervention arm, consisting of monthly one-on-one and small group sessions with a youth peer mentor (YPM) and optional caregiver support groups, or the usual care arm. The design of the Project YES! intervention was based on prior USAID-funded formative research with AYA and their caregivers (Denison et al. 2015; Denison et al. 2018; McCarraher et al. 2018; FHI 360 2013). Project YES! also drew upon previous USAID-supported work including the AIDStar Transition Toolkit (Duffy, Bergmann \& Sharer 2014) and the Positive Connections support group guide (FHI 360 2013; FHI 360 et al. 2013). To test the impact of Project YES!, survey data and blood samples were collected at baseline and at a post-intervention follow-up conducted immediately after the 6-month intervention. While the study continued after the post-intervention follow-up with a cross-over design, with all participants receiving a form of the intervention, the data presented in this report are impact data based on the baseline and post 6-month intervention follow-up data. For the impact analysis, we summarized baseline sociodemographic and clinical characteristics of the study sample using counts and percentages for categorical variables and means and standard deviations for continuous variables. Generalized estimating equation models evaluated the effect of study arm over time on viral suppression, antiretroviral treatment (ART) adherence gap, and internalized stigma. Internalized stigma was assessed as feelings of worthlessness, shame, and blame due to living with HIV. Analyses of baseline data were also conducted to estimate the prevalence of experiences of violence among HIV-positive AYA in Zambia, and in-depth interviews were carried out with AYA to explore the relationship between experiences of violence and HIV care and treatment practices. Finally, through the Project SOAR small grant mechanism, one of the Project YES! co-investigators analyzed the prevalence and characteristics of HIV drug resistance among this population of AYA living with HIV. 


\section{KEY FINDINGS}

Altogether data from 272 enrolled participants were analyzed. Key findings include:

\section{Project YES! Main study findings}

\section{Project YES! is a feasible intervention}

Almost all intervention participants (95\%) attended an orientation meeting with their health care providers (HCPs) and YPM, and the majority (74\%) attended at least five out of six one-on-one meetings with their YPM. These data support the feasibility of holding individual YPM meetings with HIV-positive AYA in a clinic context.

\section{Youth engagement suggests improvements in HIV-related outcomes among adolescents and young adults living with HIV}

Overall, VS increased significantly in both study arms (intervention arm baseline $64 \%$ to followup 73\%; comparison arm baseline $64 \%$ to follow-up $71 \%$ ) (OR 1.49, 95\% Cl 1.08-2.07) without evidence of a significant difference between study arms (Denison et al. 2020). In a stratified analysis, however, participants attending an HIV clinic at the children's hospital experienced an increase in VS by a factor of 4.7 relative to the comparison arm [interaction term OR 4.66, 95\% $\mathrm{Cl}$ 0.84-11.78], (intervention arm baseline 38\% increasing to $71 \%$; comparison arm baseline of $60 \%$ decreasing to $59 \%)$. Internalized stigma also significantly decreased in the intervention arm across both the pediatric and adult study sites by a factor of 0.39 (OR $0.39,95 \% \mathrm{Cl} 0.21-0.73$ ), 50 percent at baseline to 25 percent at follow-up (Denison et al. 2020). Based on this data, the HIV/AIDS Prevention Research Synthesis (PRS) project at the U.S. Centers for Disease Control and Prevention (CDC) has selected the Project YES! intervention to be added to the Compendium of Evidence-Based Interventions and Best Practices for HIV Prevention for Structural Interventions and Medication Adherence (inclusion forthcoming).

\section{HCP support the Project YES! approach}

HCP support is critical for Project YES! refinement and scale up. Project YES! HCPs across the four participating clinics shared how they valued the YPMs as members of their clinic staff uniquely placed to provide attention and support to AYA that the nurses-in-charge and other clinic staff simply do not have the time or resources to provide.

2 [The YPMs and I] complemented each other. They were everything I'm not. I'm not an adolescent, they're an adolescent. I'm not going through what they're going through, they're going through it. So, most of the things that I couldn't do for this peer, they were able to....

\section{AYA living with HIV experience high levels of violence}

Among HIV-positive AYA in Zambia, an estimated 72 percent of males (95\% Cl 60.3\%-81.2\%) and 75 percent of females (95\% $\mathrm{Cl} 66.6 \%-81.1 \%)$ had experienced some form of physical violence, psychological abuse, or forced sex in the past year. Some, but not all, interviewees qualitatively 
described how violence influenced their viral load (VL) (Merrill et al. 2020). For instance, several experienced psychological abuse due to their HIV status, which spurred thoughts of suicide and hampered medication adherence (Merrill et al. 2021).

\section{YPM as "game changers"}

YPMs discussed how they changed both their clients' lives and their own through Project YES! In terms of their clients, YPM discuss how they shared their struggles and role modeled positive health behaviors: "[It's] not like just talking maybe from a booklet. I'm giving myself as an example...you're helping someone from true life experiences." Additionally, YPMs' experiences resulted in powerful personal transformations in HIV-related health behaviors, conceptions of self, and plans for the future. Many expressed seeing themselves now as community leaders"ambassadors," "game changers," and "not just alone in this world." YPMs were also adamant that Project YES! should expand so other HIV-positive AYA might benefit, stating that "we just need to continue."

\section{Small grant findings on HIV drug resistance}

\section{The importance of resistance testing}

Project YES! results revealed that in this study population, three out of every four youth with viral failure have at least one HIV drug resistant (HIVDR) mutation. This finding has two key implications: a) that potentially a large proportion of youth failing may be resistant to a drug they are currently taking, and b) at least 25 percent of failing youth are failing due to reasons other than resistance, such as incomplete adherence. These factors are critical to take into consideration, particularly with the current emphasis on undetectable=untransmissible and opportunities for differentiated care to provide the support and appropriate medicines for young people with viral failure. Dr. Miti, the lead on this small grant, published these findings as a first-author peer-reviewed publication (Miti et al. 2020). In addition, the findings from Dr. Miti's analysis informed the adoption of new $\mathrm{MOH}$ guidance that HIVDR testing occur at first line failure (instead of the previous guidance to test for resistance at second line failure) as the new standard of care.

\section{CONCLUSION AND RECOMMENDATIONS}

Several recommendations come out of this research.

1. Project YES! YPMS should be incorporated into clinics as a feasible and effective way to increase viral suppression and healthy development among AYA living with HIV. As part of this recommendation, implementation science methods should inform the refinement and scale-up of the Project YES! peer mentoring approach.

2. HIV clinics need to address violence-related needs of AYA attending HIV care. Program options include screening for violence and referring to services, or hiring in-house experts to address the impact violence has on the health and HIV outcomes among this particularly vulnerable population. 
3. Youth engagement is critical to address the HIV epidemic in sub-Saharan Africa and to successfully achieve UNAIDS 95-95-95 targets. Project YES! offers a concrete evidence-based approach for engaging youth both as the implementers and as the recipients of Project YES!.

4. The impact of Project YES! in adult clinic settings and the intersection of internalized stigma and viral suppression over time need further exploration. Various biases, including the large percentages lost to follow-up in the adult settings, combined with high viral suppression rates make the findings from the adult settings inconclusive. Future iterations of Project YES! should focus on AYA with viral failure and in tracing lost clients to address these gaps.

5. Project YES! offers a concrete evidence-based approach that engages youth both as implemetners and as recipients. Project YES! was selected by the CDC's HIV/AIDS Prevention Research Synthesis Project to be included in the Compendium of Evidence-Based interventions and Best Practices for structural interventions and medication adherence, (including in Compendium website is forthcoming).

6. HIVDR testing: Project YES! baseline data provide evidence that has informed changes in $\mathrm{MOH}$ resistance testing guidance in support of HIVDR testing when first line failure occurs. HIVDR data can provide critical information for clinical ART decisions and could inform differentiated care services for AYA with viral failure who have and do not have HIVDR (Miti et al. 2020). 


\section{INTRODUCTION}

Project SOAR's comprehensive review of the literature on adolescents living with HIV in subSaharan Africa, prepared for a Technical Advisory Network (TAN) meeting, highlighted the importance of adolescents and young adults (AYA) developing the skills to manage their HIV as they transition into adulthood (Denison et al. 2016). Adolescents with chronic illnesses often struggle with adherence to medication and medical appointments, and youth living with HIV are unfortunately no different. Evidence of the challenges faced by HIV-positive youth globally is seen in their low rates of viral suppression (VS). For example, in Zambia only about one-third of 15- to 24-year-olds have achieved VS, a lower proportion than any other age group (Ministry of Health [MOH] 2019).

Internationally, there have been efforts to highlight the needs of youth and the kinds of support they require as they learn to self-manage their HIV (Pequegnat et al. 2001; Pequegnat \& Bell 2011; Pequegnat \& Szapocznik 2000; Perrino et al. 2000; Thurman, Jarabi, \& Rice 2012), For instance, PEPFAR/USAID issued guidance on the transition of care for adolescents entitled the AIDSTAR-One toolkit for transition (Duffy, Bergmann \& Sharer 2014). This guidance document emphasizes that for some adolescents and young adults, the transition of care process includes physically moving to an adult HIV care facility. Most youth though, especially in Zambia where this research was conducted, are already receiving care in an adult HIV clinic, yet still need support to develop the skills to manage their HIV. This research addresses these gaps in knowledge about how to best support adolescents and young adults transitioning to HIV self-management in the context of both child-focused and adult-focused HIV care settings. Johns Hopkins University, in partnership with the Arthur Davison Children's Hospital, implemented this study through the USAID-funded Project SOAR (led by the Population Council). The main study objectives are listed below.

\section{PROJECT YES! STUDY OBJECTIVES}

The main study objectives were to:

1. Assess the impact of a clinic-based peer mentoring approach on viral suppression and internalized stigma among youth in different care facility settings (e.g., pediatric hospital, adult hospital, and primary care facilities). Hypothesis: Youth in the intervention arm will experience greater HIV viral suppression at the end of six months of the intervention than youth in the comparison arm.

a. Conduct a stratified analysis to examine the impact of a peer mentoring approach separately among: a) the AYA participants (15-24 years) attending HIV care in a children's Hospital; and b) among AYA participants (ages 15-24 years) who are attending adult care. This will be a sub-analysis of the larger study to determine the effects of the intervention among these two distinct groups of AYA. 
2. Examine the experiences of youth and their families, health care providers (HCPs), peer mentors, and study staff with the clinic-based peer-mentoring approach through in-depth interviews (IDIs).

In addition to the main study objectives, the research team was awarded a capacity-strengthening small grant for our colleague, Dr. Sam Miti, a Project YES! co-investigator, to lead an analysis of baseline viral failure and resistance test results, and a supplemental grant to analyze baseline violence data and to conduct IDIs with youth with varying experiences of violence. The objectives related to these additional analyses using baseline data were:

\section{Small grant resistance analysis}

SG1. Estimate the prevalence of HIV treatment failure defined as $V L \geq 1,000$ copies/mL among youth aged 15 to 24 years receiving HIV care (ART) for at least six months.

SG2. Estimate the prevalence of HIV drug resistance among youth aged 15 to 24 years receiving ART for at least six months.

SG3. Determine the patterns of HIV drug resistance mutations in AYA failing HIV treatment.

\section{Supplemental grant violence analysis}

Supp1. Estimate the prevalence and perpetrators of violence against AYA living with HIV in Ndola, Zambia using baseline reports of physical and sexual violence and psychological abuse.

Supp2. Examine associations between past-year experiences of violence and viral load failure among HIV-positive AYA using baseline data.

Supp3. Qualitatively explore the relationship between experiences of violence and engagement in HIV care and treatment among Project YES! AYA participants. 


\section{METHODOLOGY}

\section{STUDY DESIGN}

The study was designed to test the impact of the Project YES! clinic-based youth peer mentoring program on HIV viral suppression, adherence, and internalized stigma, through a randomized controlled trial (RCT). This RCT was conducted in four HIV clinics in Ndola Zambia, including one children's hospital, one adult hospital, and two primary care facilities, among HIV-positive adolescents and young adults ages 15 to 24 years. After completing a baseline survey and blood draw, the consecutive sample of participants were randomly assigned to either the intervention or comparison arm. After completion of the first Project YES! intervention phase that lasted approximately six months, the participants completed the follow-up data collection. For this impact analysis, we analyzed the findings from baseline and follow-up survey and a blood draw for VL testing. Resistance testing was conducted at baseline for those participants who had VL failure (defined as 1,000 copies/mL or higher). As the surveys contained questions about violence and thoughts of suicide, the study also developed a safety protocol, so data collectors and youth peer mentors (YPMs) clearly knew to refer AYA participants who appeared distressed or had experiences of severe physical or any sexual violence to the designated HCPs at the clinic.

After the follow-up data collection was completed, our study team began conducting qualitative IDIs with participants, their caregivers, and the HCPs, peer mentors, and study staff involved in delivering the program to explore their experiences with Project YES! Qualitative IDIs were also conducted with youth with experiences of violence to address the supplemental analysis objectives.

\section{DESCRIPTION OF INTERVENTION}

\section{Project YES! intervention arm}

The Project YES! YPMs were HIV-positive young adults ranging in age from 21 to 26 years who were identified by HCPs. These YPMs underwent a capacity-building process that included an initial two-week training, a midterm in-service training, and supportive supervision throughout the program. This process equipped YPMs with the skills to mentor AYA participants based on their shared experience of living with HIV. YPMs were then placed in clinics as staff in the lay health worker category, earning 2,368 Kwacha per month (around US $\$ 150$ at the time). AYA participants had the opportunity to meet with their YPMs seven times. The first meeting was an orientation meeting with an HCP, their assigned YPM, and an adult caregiver (if invited by the youth participant). During the orientation meeting, the participant's viral load test result was shared and the purpose of Project YES! to help youth either maintain or lower their viral count was reviewed. The remaining six meetings were monthly one-on-one meetings between the YPM and youth participant. Participants were also invited to monthly youth group meetings facilitated by YPMs, 
with an HCP invited to attend when clinical or technical information was needed. Adult caregivers of youth participants were also offered a total of three caregiver group meetings, facilitated by an HCP and held every other month, to help caregivers support their AYA living with HIV.

The project also provided Youth and Caregiver Journals that participants could use to track adherence, engage with educational topics, and reflect on their journeys. These journals drew upon the USAID-funded AIDSTAR Transition Toolkit and the Positive Connections Youth Group Manual (Duffy, Bergmann \& Sharer 2014; FHI 360 et al. 2013).

In the children's hospital clinical setting, the study team also worked with the study clinician and the clinic HCPs to assess AYA participants for a physical move from the children's pediatric HIV clinic to an adult HIV clinic. Participants who were eligible to be transferred, based on clinical and social factors, were invited to attend a group transition meeting prior to the start of the intervention to tour the adult clinic. Transitioned youth then participated in intervention activitiesincluding meeting with their YPM and youth group-and received their clinical care in the adult clinic.

\section{Comparison arm}

Participants in the comparison arm received the standard of care for AYA as offered at the HIV clinics, including regular clinic visits and the option of joining monthly youth group meetings. After the follow-up data collection, comparison arm participants started the Project YES! intervention as described above, including transitioning eligible youth from the children's hospital to the HIV clinic in the adult hospital.

\section{DATA COLLECTION METHODS}

Consecutively sampled youth who met the eligibility criteria underwent the informed consent process. In accordance with Zambian law, participants aged 15 to 17 years provided written assent and their parent/guardian provided parental consent (Government of Zambia 2013). Eligible youth were between the ages of 15 and 24 years, aware of their HIV status, had been on ART for six or more months, spoke Bemba or English, and planned to be available to attend study activities over the next 18 months as needed. Exclusion criteria for youth included being too sick to participate, attending boarding school, having a sibling already enrolled in the study (one youth per household), or having participated in a prior adolescent/caregiver intervention study held at two of the study sites (FHI 360 2013).

After baseline data collection, all participants were randomized to either the intervention or the comparison arms. Randomization was stratified by sex and age within each of the four clinics for a total of 16 strata. Baseline and post-intervention follow-up surveys were administered by trained research assistants using Magpi data collection tablet-based software. As part of the safety protocol, research assistants referred participants who reported severe violence or thoughts of suicide in the past week to HCPs for additional care. Data were also abstracted from participants medical charts including ART start-date and pre-ART CD4 cell count. 
Baseline and follow-up HIV-1 RNA viral load testing was conducted using the CobusAmpli-prep/ CobusTaqman 96 machine (Roche Systems, Germany). Baseline blood samples with viral loads of 1,000 copies or more per milliliter (virologic treatment failure) were further subjected to an HIV drug resistance test using an Applied Biosystems Genetic Analyzer model 3500XL (Hitachi, Japan).

Qualitative IDIs were conducted after the follow-up data collection was completed. To understand experiences with Project YES!, intervention arm youth participants were purposively selected and interviews were conducted with AYA clients, their caregivers, the HCPs, peer mentors, and study staff. To understand experiences of violence, AYA clients from both study arms were purposively selected. Trained interviewers conducted IDIs in English or Bemba in private locations at study clinics, which were audio-recorded, translated (when applicable), and transcribed.

\section{ETHICAL REVIEW}

This study was reviewed and approved by the ERES Converge Institutional Review Board in Zambia, the Zambia MOH through the National Health Research Authority, and the Johns Hopkins Bloomberg School of Public Health Institutional Review Board in the United States. Participants were reimbursed $50 \mathrm{Kwacha}$ (about US\$5.00) for their time and travel to the clinic for studyrelated appointments. In addition, intervention participants were provided snacks during monthly group meetings.

\section{DATA ANALYSIS}

For the first objective, to test the impact of Project YES! on AYA HIV-related outcomes, we summarized baseline sociodemographic and clinical characteristics of the study sample using counts and percentages for categorical variables and means and standard deviations for continuous variables. Generalized estimating equation models evaluated the effect of study arm over time on the pre-determined outcomes for the primary analysis of viral suppression, antiretroviral treatment (ART) adherence gap, and internalized stigma. These data have been published in Project SOAR's PLOS Collection (Denison et al. 2020). VS was defined as $<1,000$ copies/mL.

Two questions were asked of participants to measure ART adherence treatment gap of 48 or more consecutive hours: "In the past three months, did you have a day when you did not take any $A R T$ drugs?" and "What were the most days in a row that you missed swallowing your drugs in the past three months?" A participant was considered to not have a treatment gap if they said no to missing any full days of ART drugs in the past three months, or if they said yes and only missed one day. Otherwise, they were considered to have a treatment gap of 48 consecutive hours or more.

The internalized stigma variable was measured using three agree/disagree questions from the Internalized AIDS Stigma Scale (IA-RSS) (Kalichman et al. 2009), that have been used in a previous study among a population of HIV-positive adolescents in Ndola, Zambia (Denison et al. 
2018). This measure asks participants to either "agree" or "disagree" with each of the following three statements: (1) You feel guilty that you are HIV-positive; (2) You are ashamed that you are HIV-positive; and (3) You sometimes feel worthless because you are HIV-positive. From these data, a binary outcome was generated with " 1 " indicating that the participant answered "agree" to at least two of the three questions, and "0" otherwise (Denison et al. 2018).

We evaluated the level of intervention exposure as the number of one-on-one peer mentoring sessions and the number of group sessions each youth attended, as well as if the youth had a caregiver attend any of the Project YES! sessions. We conducted chi-square tests for the null hypotheses that attending at least five out of six group meetings or having a caregiver attend any of the meetings was associated with VS at follow-up.

For the qualitative analysis, all IDIs were translated and transcribed, checked for accuracy, summarized in memos, and coded for key themes using Nvivo qualitative analysis software.

For the small grant on resistance among HIV-positive youth, cross-sectional analyses were conducted using baseline RCT viral load and resistance test results. Blood samples for HIV viral load testing were obtained and collected in Ethylenediamine tetraacetic acid (EDTA) anticoagulated bottles. HIV viral load was determined using the Cobas Ampliprep/CobasTaqman 96 machine (Roche Systems, Germany). Blood samples with a viral load of 1,000 copies or more per milliliter (virologic treatment failure) were further subjected to an HIV drug resistance test using an Applied Biosystems Genetic Analyzer model 3500XL (Hitachi, Japan). HIV-1 RNA was extracted from 140 milliliter of plasma using the Qiagene QiAmp viral RNA mini kit (QIAGEN, Germany). Genotyping encompassed protease and codons 1-230 of reverse-transcriptase, using Applied Biosystems HIV-1 Genotyping: Cycle sequencing module. Sequences were assembled and manually edited using Sequencer version 4.5 software (Gene codes, Ann Arbor, MI). The outcomes of the HIV resistance tests were reported using the Stanford HIV drug resistance database. ${ }^{1}$ HIVDR was defined as the presence of at least one drug resistance-associated mutation according to the World Health Organizations (WHO) 2009 Surveillance Drug Resistance Mutation list, using the Stanford Calibrated Population Resistance analysis tool (version 4.1 beta). ${ }^{1}$ We conducted a cross-sectional analysis of all baseline HIVDR results to determine HIVDR prevalence and characteristics among adolescents and young adults with virologic treatment failure.

For the supplemental grant on violence, cross-sectional analyses were conducted using baseline RCT data. Lifetime and past-year prevalence of physical violence, psychological abuse, and forced sex were estimated. These estimates were weighted according to the age and sex distributions of HIV-positive males and females, ages 15-19 and 20-24 years, in Zambia, using the 201314 Zambia Demographic and Health Survey. This weighting was used to generate prevalence estimates representative of HIV-positive AYA in Zambia. Self-reported measures of experiences of violence were adapted from the IPSCAN Child Abuse Screening Tool (ICAST) (ISPCAN 2006) and the World Health Organization (WHO) Multi-Country Study on Women's Health and Domestic Violence against Women (WHO MCS) (WHO 2005).

The team has also started the coding and analysis of the 41 qualitative IDIs with Project YES! youth who have experienced varying degrees of violence.

${ }^{1}$ http://hivdb.stanford.edu/ 


\section{KEY FINDINGS}

\section{CHARACTERISTICS OF THE PARTICIPANTS}

Out of the 276 enrolled, three participants were excluded from analyses because they had been on ART for less than six months, bringing the analysis sample to 273.

Figure 1 CONSORT flow diagram

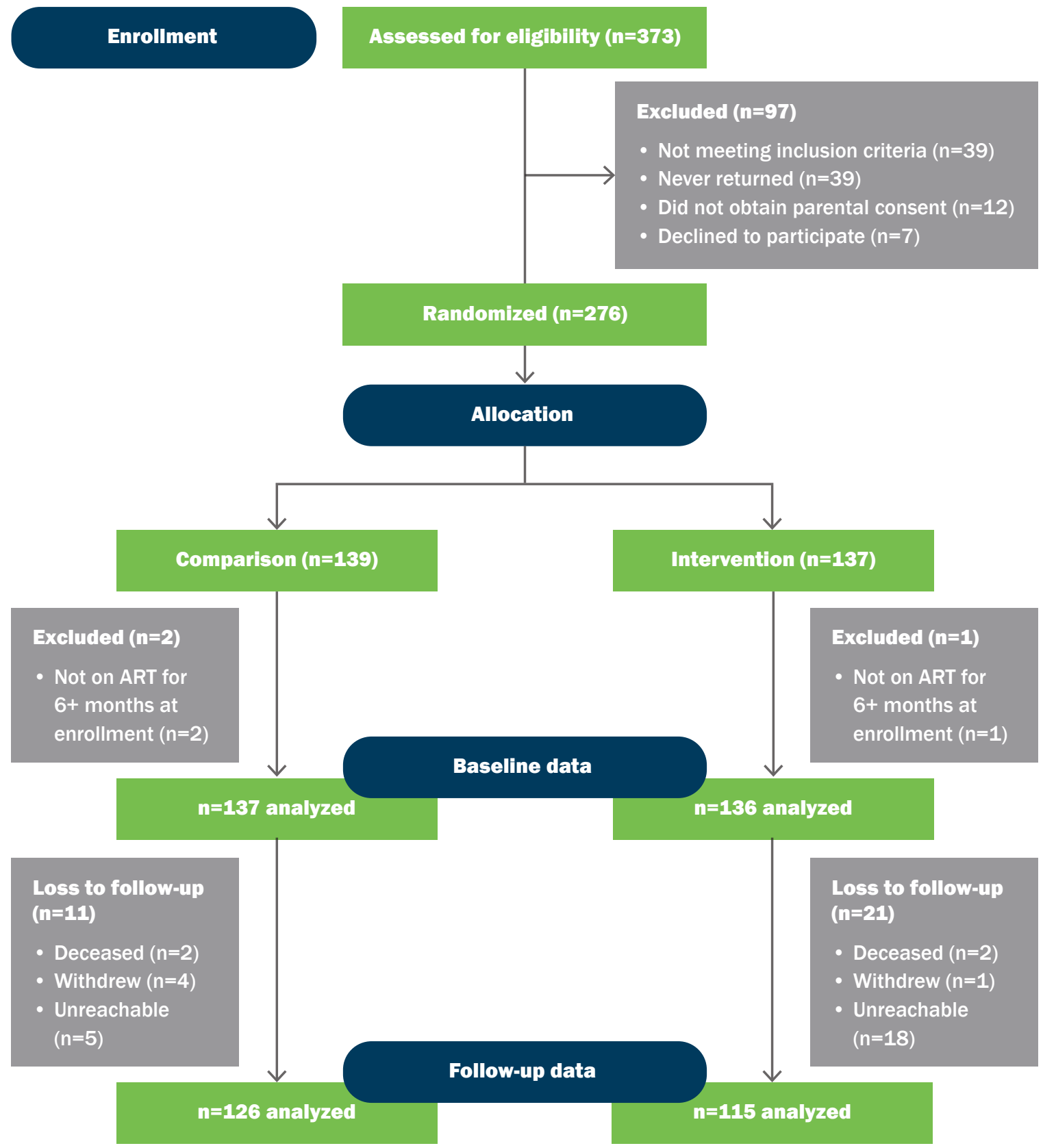

Published in Denison et al. 2020 
At baseline, the average age was 19 years (range 15-24), and more than half (59\%) were females (Table 1). Almost three-quarters self-reported having acquired HIV perinatally (73\%).

Project YES!, as part of the program, also physically transitioned 23 consenting participants with VS and no known psychosocial issues from the children's hospital to the adult hospital clinic.

Table 1 Baseline sociodemographics of youth participants

\begin{tabular}{|c|c|c|c|}
\hline & Intervention (\%) & Comparison (\%) & Total (\%) \\
\hline Participants & $137(50.18)$ & $136(49.82)$ & $273(100.00)$ \\
\hline Mean age in years (range) & $19.12(15-24)$ & $19.10(15-24)$ & $19.11(15-24)$ \\
\hline \multicolumn{4}{|l|}{ Age } \\
\hline $15-19$ years old & $87(63.50)$ & 87 (63.97) & $174(63.74)$ \\
\hline $20-24$ years old & $50(36.50)$ & 49 (36.03) & $99(36.26)$ \\
\hline Missing & - & - & - \\
\hline \multicolumn{4}{|l|}{ Sex } \\
\hline Female & $82(59.85)$ & $80(58.82)$ & $162(59.34)$ \\
\hline Male & $55(40.15)$ & $56(41.18)$ & $111(40.66)$ \\
\hline Missing & - & - & - \\
\hline \multicolumn{4}{|l|}{ Primary school } \\
\hline Did not complete primary school & $20(14.60)$ & $11(8.09)$ & $31(11.36)$ \\
\hline Completed primary school & $117(85.40)$ & $123(90.44)$ & $240(87.91)$ \\
\hline Missing & - & $2(1.47)$ & $2(0.73)$ \\
\hline \multicolumn{4}{|l|}{ How acquired HIV } \\
\hline From parents & $97(70.80)$ & $101(74.26)$ & $198(72.53)$ \\
\hline Through sex & $11(8.03)$ & $16(11.76)$ & 27 (9.89) \\
\hline Another way & $10(7.30)$ & $4(2.94)$ & $14(5.13)$ \\
\hline Don't know & $18(13.14)$ & $14(10.29)$ & $32(11.72)$ \\
\hline Missing & $1(0.73)$ & $1(0.74)$ & $2(0.73)$ \\
\hline \multicolumn{4}{|l|}{ Baseline VL suppression } \\
\hline (<1,000 copies/mL) & $87(63.50)$ & $86(63.24)$ & $173(63.37)$ \\
\hline Missing & - & $1(0.74)$ & $1(0.37)$ \\
\hline
\end{tabular}

\section{OBJECTIVE 1 AND 1A: PRIMARY ANALYSIS ON PROJECT YES! IMPACT AND STRATIFIED ANALYSES}

\section{Objective 1: Overall impact}

Almost all AYA in the intervention arm (95\%) attended an orientation meeting with the YPM, HCP and, if invited by the youth, their caregiver. Seventy-four percent also attended at least five of the six YPM one-on-one meetings. As published in Denison et al. (2020), VS significantly increased in both study arms (OR 1.49, 95\% Cl 1.08-2.07), from 64 percent to 73 percent and 64 percent to 71 percent in the intervention and comparison arms, respectively (Figure 2). Please refer to the Aim 1 a results further below to see the statistically significant stratified VS results. 
Figure 2 Viral suppression at baseline and follow-up by study arm

GEE results adjusted for sex, age, and clinic:

Intervention vs. comparison arm effect (overall): OR 1.03 (95\% Cl 0.68-1.57)

Time effect (overall): OR 1.49 (95\% Cl 1.08-2.07)

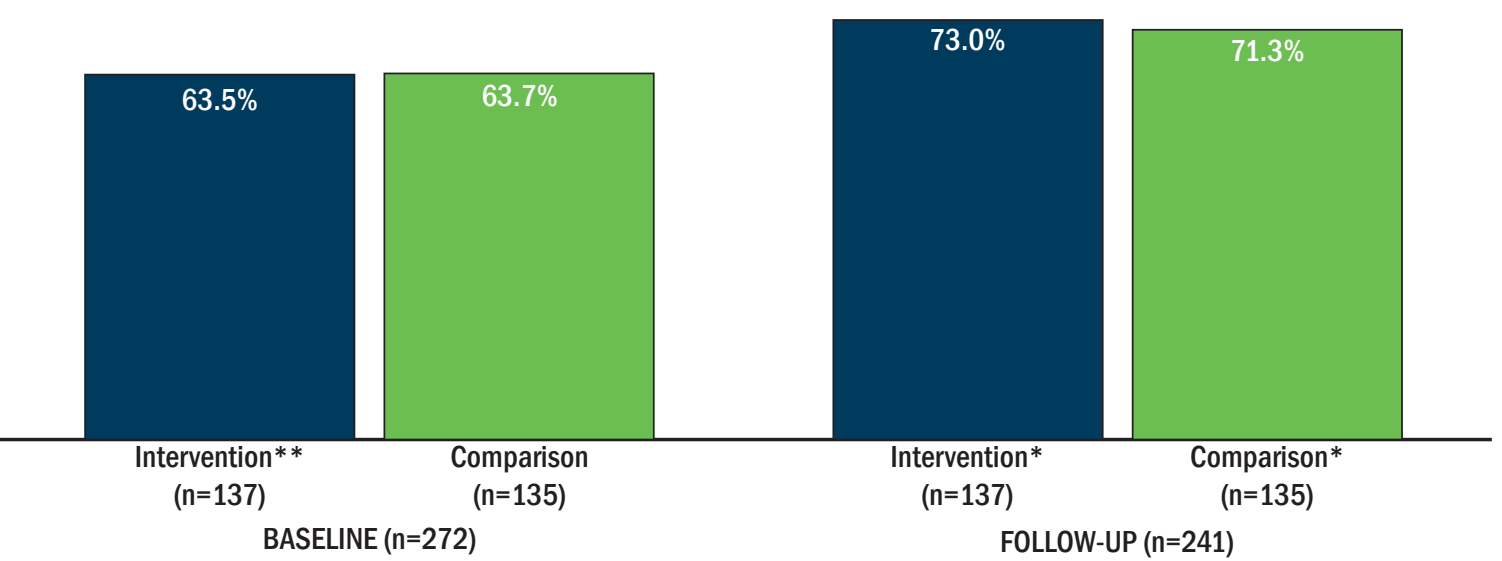

**Missing baseline for VL for 1 participant; *11 (8.03\%) lost to follow-up in intervention arm; 20 (14.81\% lost to follow-up in comparison arm

Published in Denison et al. 2020

In terms of ART adherence treatment gap, the intervention arm went from 45 percent selfreporting a treatment gap at baseline down to 34 percent at follow-up, while the comparison arm increased slightly from 33 percent at baseline to 34 percent at follow-up.

The GEE model results found that the odds of having a treatment gap were significantly higher at baseline among the intervention versus the comparison arm participants (OR:1.74, 95\% $\mathrm{Cl} 1.06$ 2.86). While the treatment gap did not statistically change from baseline to the follow-up in the comparison group (OR:1.05, 95\% $\mathrm{Cl} 0.68-1.61)$, there was a relative decrease in the intervention arm (OR interaction term: 0.63, 95\% Cl 0.35-1.13) (Denison et al. 2020)

\section{Figure 3 Treatment gap at baseline and follow-up by study arm (reported by participants)}

\section{GEE results adjusted for sex, age, and clinic:}

Intervention vs. comparison arm (baseline): OR 1.74 (95\% CI 1.06-2.86)

Time effect on the comparison arm: OR 1.05 (95\% $\mathrm{Cl} 0.68-1.61)$

Study arm x time point: OR $0.63(95 \% \mathrm{Cl} 0.35-1.13)$

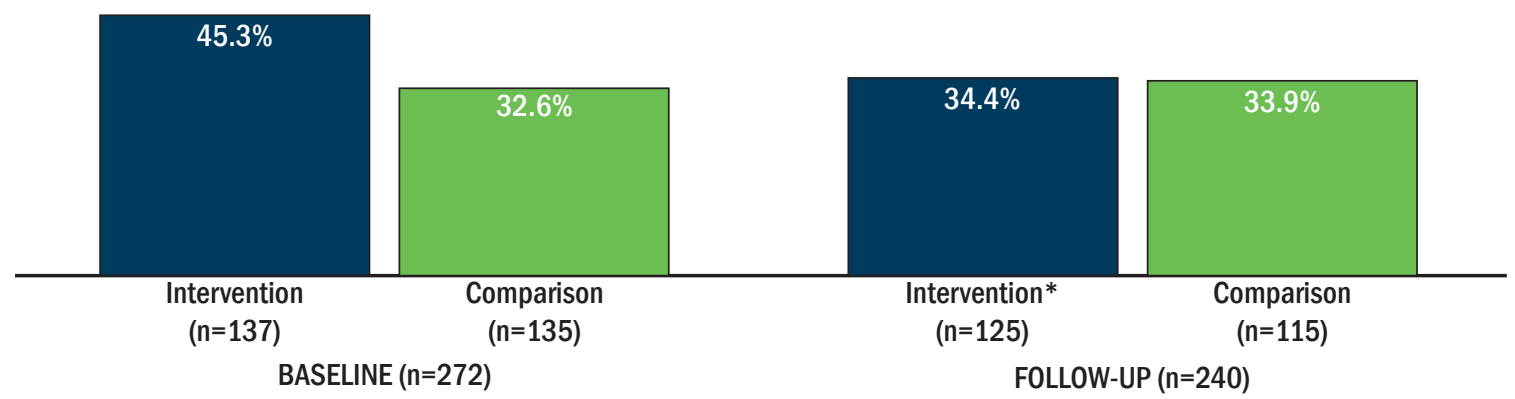

*12 (8.76\%) missing in intervention arm (11 lost to follow-up, 1 responded “don’t know’ at midline); 20 (14.81\% lost to follow-up in comparison arm

Published in Denison et al. 2020 
Internalized stigma significantly decreased in the intervention arm across all study sites $(50 \%$ at baseline to $25 \%$ at follow-up). By follow-up, internalized stigma was significantly reduced by a factor of 0.39 (OR $0.39,95 \% \mathrm{Cl} 0.21-0.73$ ) in the intervention arm relative to the reduction in the comparison arm (Figure 4) (Denison et al. 2020).

\section{Figure 4 Internalized stigma at baseline and follow-up by study arm}

GEE results adjusted for sex, age, and clinic:

Intervention vs. comparison arm effect (baseline): OR 1.22 (95\% $\mathrm{Cl} 0.75-2.01)$

Time effect on the comparison arm: OR 0.83 (95\% Cl 0.54-1.29)

Study arm x time point: OR $0.39(95 \% \mathrm{Cl} 0.21-0.73)$

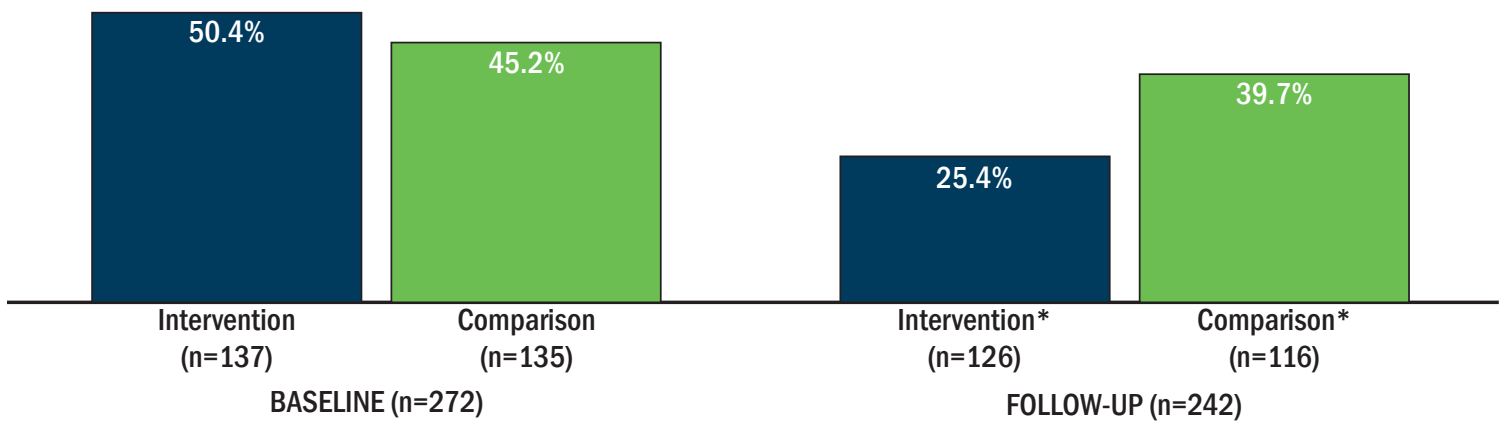

*11 $(8.03 \%)$ missing in intervention arm; 19 (14.07\% lost to follow-up in comparison arm

Published in Denison et al. 2020

\section{Objective 1a: Stratified analysis (pediatric and adult HIV clinic settings)}

As shown in Figure 5, in a stratified analysis, the children's hospital intervention participants versus the comparison participants experienced a relative increase in the odds of VS by a factor of 4.7 (interaction term OR 4.66, 95\% Cl 1.84-11.78) (Denison et al. 2020).

\section{Figure 5 Viral suppression at baseline and follow-up: Pediatric clinic only}

\section{GEE results adjusted for sex and age:}

Intervention vs. comparison arm (baseline): OR 0.36 (95\% $\mathrm{Cl} 0.17-0.79)$

Time effect on the comparison arm: OR 0.91 (95\% Cl 0.52-1.62)

Study arm x time point: OR 4.66 (95\% Cl 1.84-11.78)

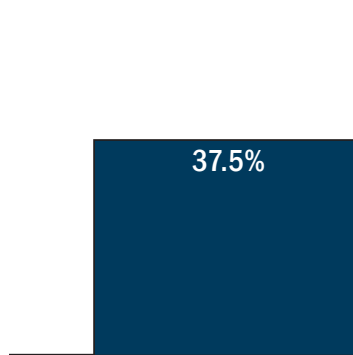

Intervention $(n=48)$

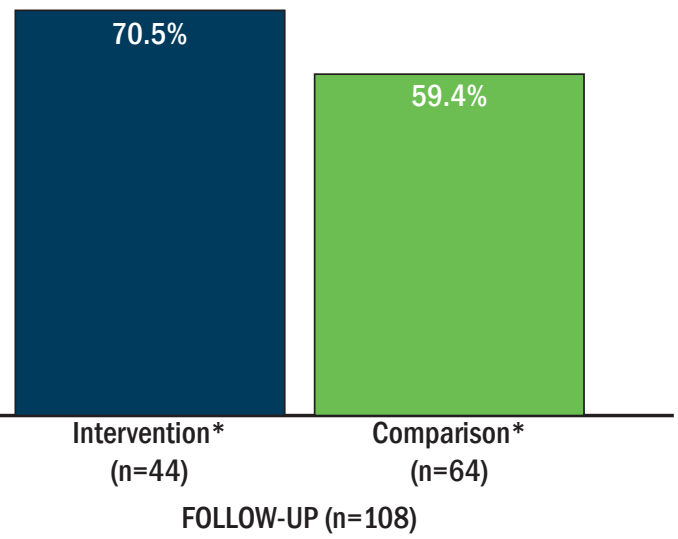

FOLLOW-UP $(n=108)$

*4 (8.33\%) missing in pediatric clinic intervention arm; 9 (12.33\%) missing in pediatric clinic comparison arm

Published in Denison et al. 2020 
In the adult clinic settings, VS at baseline in the intervention arm was 78 percent at baseline and 74 percent at follow-up. In the adult comparison arm VL suppression was 68 percent at baseline and 86 percent at follow-up (Figure 6). The GEE model for the adult sites showed that among the comparison arm participants, time had a significant effect, such that the odds of VS at followup were three times higher than at baseline (OR 2.99, 95\% Cl 1.27-7.04). Furthermore, among the intervention arm participants, there was a significant relative reduction in the odds of VS by follow-up compared to the comparison arm participants (interaction OR 0.27, 95\% $\mathrm{Cl} 0.10-0.79$ ) (Denison et al. 2020).

\section{Figure 6 Viral suppression at baseline and follow-up: Adult clinic only}

GEE results adjusted for sex and clinic: Intervention vs. comparison arm (baseline): OR 1.71 (95\% $\mathrm{Cl} 0.82-3.56)$

Time effect on the comparison arm: OR 2.99 (95\% CI 1.27-7.04)

Study arm x time point: OR 0.27 (95\% Cl 0.10-0.79)

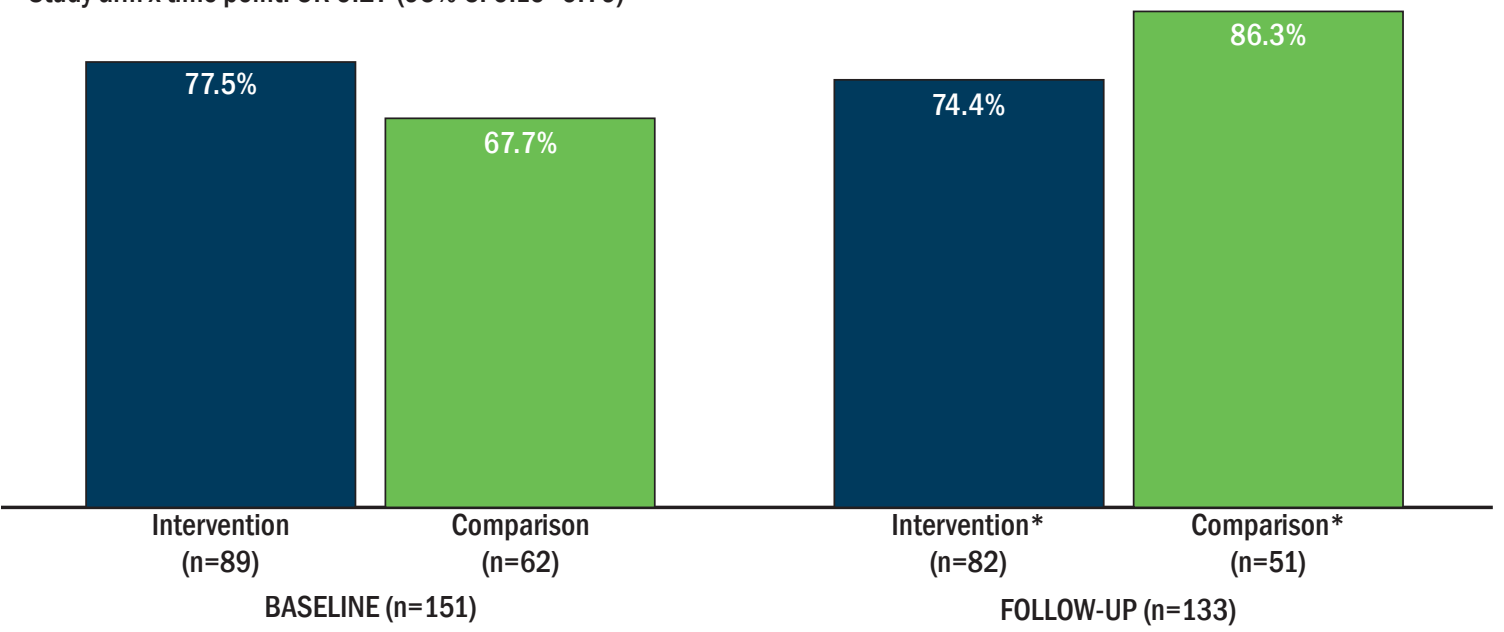

*7 (7.87\%) missing in adult clinic intervention arm; 11 (17.74\%) missing in adult clinic comparison arm

\section{OBJECTIVE 2: QUALITATIVE ANALYSES OF YPM, HCP, AYA, AND CAREGIVER IDIS}

At the time of this report, all qualitative interviews had been translated and transcribed. Coding had begun with the 41 IDIs with youth participants. Initial coding had been completed for the IDI data with the 8 YPM and the $10 \mathrm{HCPs}$. Below are preliminary results pertaining to the YPM and the HCP perspectives and experiences implementing Project YES!

\section{YPM IDIs}

IDIs with the eight YPMs who implemented Project YES! showed that YPMs connected with both male and female AYA clients by discussing their shared struggles, role modeling positive health behaviors, and establishing confidential, judgement-free environments for clients. 
2 [It's] not like just talking maybe from a booklet. l'm giving myself as an example... you're helping someone from true life experiences.

Additionally, YPMs' experiences resulted in powerful personal transformations in HIV-related health behaviors, conceptions of self, and plans for the future. Many expressed seeing themselves now as community leaders, specifically "ambassadors," "game changers," and "not just alone in this world." They described moving from feeling hopeless about their futures to excited and confident about reaching personal and professional goals:

63 I also have the right to think ahead and think about my future.

YPMs were also adamant that Project YES! should expand so other HIV-positive AYA might benefit, stating that "we just need to continue."

\section{HCP IDIs}

IDIs with $10 \mathrm{HCPs}$ showed that HCPs had an overall positive assessment of Project YES!. HCPs also felt YPMs were useful in the clinic and uniquely positioned to discuss sensitive health topics with AYA, like adherence challenges and sexual and reproductive health. As one HCP stated:

2 [The YPMs and I] complemented each other. They were everything I'm not. I'm not an adolescent, they're an adolescent. l'm not going through what they're going through, they're going through it. So, most of the things that I couldn't do for this peer, they were able to....

Prior to Project YES!, HCPs reported not fully recognizing intersectional challenges HIV-positive AYA encounter, particularly violence experienced by both female and male participants.

2 Some of the patients that I thought had no problems, had no issues, I've just realized that you don't take it for granted.... So the peer mentors have actually done a very good job because they ask so many questions and they are the ones that have opened my eyes to know that these adolescents do not only require ARVs and adherence counseling. They need to be asked a lot of questions so that you get to understand where they are, how they're feeling, what problems they're going through.

HCPs discussed ways they overcame logistical challenges to implementation, like time and space limitations, and expressed wanting more involvement in Project YES! decisions related to clinical care. They felt caregivers valued the opportunity to discuss sensitive topics during group meetings and recommended increasing caregiver involvement in future programs.

\section{SMALL GRANT RESISTANCE ANALYSIS}

Dr. Miti successfully worked with the Project YES! study team and mentors in the U.S. and Zambia to clean and analyze the baseline viral test and resistance test results. Dr. Miti has presented 
these findings during the dissemination meetings and during $\mathrm{MOH}$-sponsored meetings in Zambia. He also presented these results during the 2019 ICASA in Rwanda and and published a peer-reviewed manuscript with detailed methods and results (Miti et al. 2020).

Overall, low proportions of HIV-positive youth had viral load suppression ( $<1,000$ copies/ $\mathrm{mL}$ ). A total of 99 out of 272 analyzed youth had VL failure, of whom 77 had successful HIVDR amplification and were analyzed. Out of the 77,75 percent $(n=58)$ had the presence of at least one drug resistant mutation, indicating that at least 25 percent of youth living with HIV with VL failure are failing due to other reasons, such as incomplete adherence. Figure 7 details these study findings.

\section{Figure 7 VL failure and HIV drug resistance}

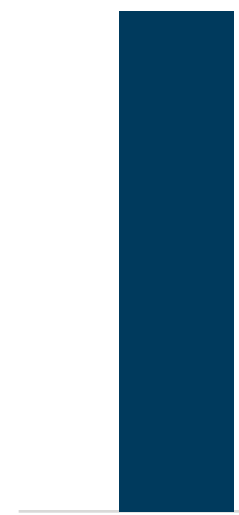

YLHIV

$(n=273)$

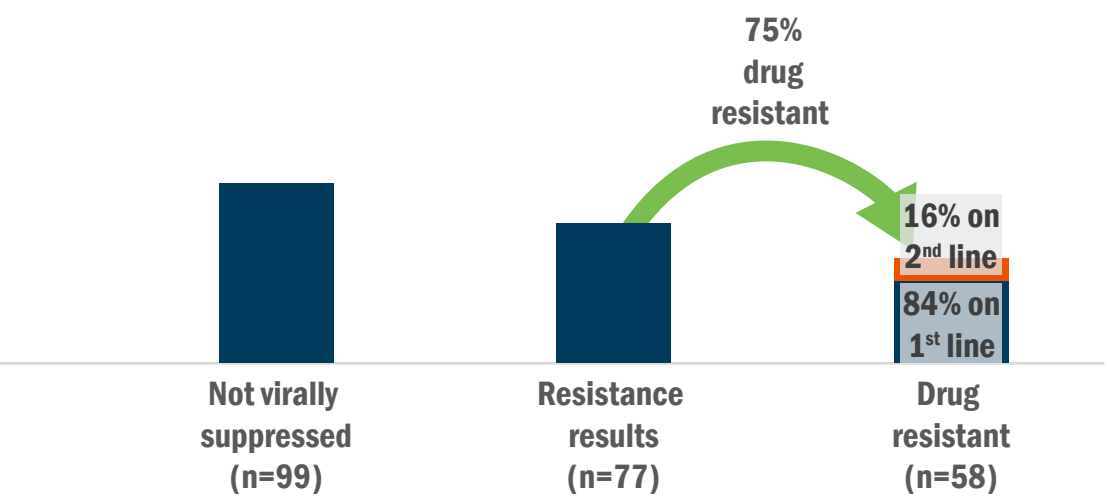

$(n=99)$ results
$(\mathrm{n}=77)$

$(n=58)$

\section{SUPPLEMENTAL VIOLENCE ANALYSIS}

The supplemental analysis on violence included 272 AYA, of whom 59 percent were female and 71 percent were self-reported perinatally infected. These quantitative and qualitative data on violence have been published in three peer-reviewed manuscripts (Merrill et al. 2020a; Merrill et al. 2020b; Merrill et al. 2021). The estimated prevalence of any type of violence experience was 78 percent (95\% Cl 72.0\%-83.4\%) for lifetime and 74 percent (95\% Cl 67.0\%-79.1\%) for past-year reports (see Table 2). Almost half (46\%, 95\% Cl 39.4\%-52.9\%) had ever experienced polyvictimization, defined as experiencing more than one type of violence. No differences in the overall prevalence of violence were observed by sex. 
Table 2 Estimated lifetime and past-year prevalence of experiences of violence among adolescents and young adults living with HIV in Ndola, Zambia $(\mathrm{n}=272)$

\begin{tabular}{|c|c|c|c|c|c|}
\hline & & & Past yea & r, stratified by sex & \\
\hline & $\begin{array}{l}\text { Lifetime } \\
\%(95 \% \mathrm{Cl})\end{array}$ & $\begin{array}{l}\text { Past year } \\
\%(95 \% \mathrm{CI})\end{array}$ & $\begin{array}{c}\text { Male }(n=108) \\
\%(95 \% \mathrm{Cl})\end{array}$ & $\begin{array}{c}\text { Female }(n=163) \\
\%(95 \% \mathrm{Cl})\end{array}$ & $p$ value \\
\hline $\begin{array}{l}\text { Any violence } \\
\text { (physical violence, } \\
\text { psychological abuse, } \\
\text { or forced sex) }\end{array}$ & $78.2(72.0-83.4)$ & $73.5(67.0-79.1)$ & $72.0(60.3-81.2)$ & $74.5(66.6-81.1)$ & 0.69 \\
\hline Polyvictimization & & & & & \\
\hline No violence & $21.8(16.6-28.0)$ & $26.5(20.9-33.0)$ & $28.1(18.8-39.7)$ & $25.5(18.9-33.4)$ & \\
\hline 1 type & $32.2(26.2-38.8)$ & $35.7(29.4-42.6)$ & $33.4(23.6-44.8)$ & $37.3(29.4-45.9)$ & 0.84 \\
\hline $2+$ types & $46.1(39.4-52.9)$ & $37.8(31.4-44.5)$ & $38.6(28.3-50.0)$ & $37.2(29.5-45.7)$ & \\
\hline
\end{tabular}

Notes: $\mathrm{n}$ and percentages are weighted; $\%$ are column percentages and may not add up to 100 , since participants could select more than one form of violence. $p$ values are from $F$ tests.

Preliminary findings from IDIs with youth who reported experiences of violence show that some but not all interviewees described how violence influenced their VL. For instance, several experienced psychological abuse due to their HIV status, which spurred thoughts of suicide and hampered medication adherence:

2 My being HIV-positive has brought me trouble at this home...Each time they [insult and ridicule me], I just think of killing myself. I even stop[ped] taking my medication... for two months.

Analyses of the violence data were ongoing at the time of this report. Planned analyses of the quantitative data include examining who the perpetrators of violence among youth are and assessing the association between experiences of violence and VL failure among youth. For the qualitative data with 41 youth, we will analyze the data to better understand how violence effects HIV-positive young people and their self-management of their HIV. 


\section{DISCUSSION}

\section{PROJECT YES! IMPACT}

This study provides critical data on a youth-led approach to improving HIV outcomes among adolescents and young adults in Zambia. The YPMs, with quality training, practice, and pay, became valued members of the health care system and were able to identify with, and provide concrete support to, AYA who were coping with their HIV status on a day-to-day basis.

The overall participation rates of intervention participants provide concrete evidence that Project YES! is a feasible model for engaging and working with HIV-positive AYA. Further, the data show that VS increased among AYA in pediatric settings and feelings of internalized stigma decreased across AYA in all settings (adult and pediatric). There are little data out there on what impacts VS and internalized stigma, so these findings are of particular importance, especially as one's sense of self solidifies during adolescence (WHO 2014), so reducing internalized stigma is a noteworthy success.

Not only did the YPMs impact their clients' lives, but they also very eloquently describe how Project YES! impacted their own sense of self-worth and their plans for the future. During the dissemination meeting held in Lusaka, YPMs and youth clients who were hesitant to disclose their status to others in the past freely shared their personal experiences with stakeholders and strangers. One workshop participant from the HIV/AIDS Program at the University Teaching Hospital remarked on how professionally and confidently the YPM carried themselves, suggesting they were exceptional youth living with HIV. While the YPM in Project YES! have exceeded expectations and continue to impress and teach us, their success is evidence of the potential transformation that all youth living with HIV might achieve when provided with guidance, respect, and opportunity. These empowered voices can strengthen and shape our response to the HIV epidemic.

Another key finding is HCP support for Project YES! A clinic-based program cannot succeed without the endorsement and active engagement of its clinical providers, and Project YES! HCPs clearly articulated how much they valued the YPMs in their clinics, while also emphasizing the need for increased communication and more caregiver involvement. These findings correspond with the Positive Youth Development approach, which emphasizes the importance of adult connections in supporting healthy adolescent development (Alvarado et al. 2017; Lerner RM 2009; Lerner et al. 2006). HCP feedback and support is essential to refine and scale-up the Project YES! approach and future implementation should test innovative ways to further engage more caregivers in the process.

Overall, Project YES! was well attended, so much so that it was not possible to conduct an exposure analysis to determine if the one-on-one versus group meetings were related to greater odds of VS, as there was little variability in attendance. These high rates of attendance, coupled 
with decreased internalized stigma and increased VS among pediatric clients, suggest the feasibility and value of the program and the potential for scale-up.

\section{THE INTERSECTION OF VIOLENCE AND VIRAL SUPPRESSION}

This study also highlights the overwhelming prevalence of violence that AYA living with HIV in Zambia face. While experiences of violence are a known predictor of poorer HIV-related outcomes among adult females, this study is the first to our knowledge in Zambia to examine violence among AYAs. Violence is a challenging issue that must be addressed in this population, given that an estimated three-quarters of youth experienced violence in the past year. Notably, youth in this study were recruited as they attended HIV clinics, suggesting an underutilized opportunity to identify and support youth experiencing violence who are already accessing the health system. Violence care and prevention need to be integrated into HIV services, either directly in the clinic or through referrals, as experiences of violence are undermining the potential impact of ART to improve AYA health and to achieve the UNAIDS 95-95-95 targets (UNAIDS 2014).

\section{LIMITATIONS}

There are limitations to this study that need to be taken into consideration. First, the loss to follow up differed between the adult and pediatric clinic settings, with greater proportions of youth lost from the adult sites. This bias reinforces the need for further research among youth in adult settings around retention and the potential impact of Project YES! on VS. Another limitation related to the violence analyses is the cross-sectional approach that identifies associations but cannot determine causality. Finally, there are also measurement issues to consider, with social desirability biases perhaps limiting AYA's self-reports of experiences of violence. 


\section{CONCLUSION AND RECOMMENDATIONS}

\section{CONCLUSIONS}

\section{Project YES! main study findings}

\section{Project YES! is a feasible intervention}

Almost all intervention participants (95\%) attended an orientation meeting with their HCPs and YPM, and the majority (74\%) attended at least five out of six one-on-one meetings with their YPM. These data support the feasibility of holding individual YPM meetings with HIV-positive AYA in a clinic context.

\section{Youth engagement leads to improvements in HIV-related outcomes among adolescents and young adults living with HIV}

Overall, VS increased significantly in both study arms (intervention arm baseline $64 \%$ to followup $73 \%$; comparison arm baseline $64 \%$ to follow-up 71\%; OR 1.49, 95\% Cl 1.08-2.07) without evidence of a significant difference between study arms (Denison et al. 2020). In a stratified analysis, however, participants attending an HIV clinic at the children's hospital experienced an increase in VS by a factor of 4.7 relative to the comparison arm (interaction term OR 4.66, 95\% Cl.84-11.78; intervention arm baseline $38 \%$ increasing to $71 \%$; comparison arm baseline of $60 \%$ decreasing to $59 \%)$. Internalized stigma also significantly decreased in the intervention arm across both the pediatric and adult study sites by a factor of 0.39 (OR 0.39, 95\% Cl 0.21-0.73; $50 \%$ at baseline to $25 \%$ at follow-up). Based on this data, the HIV/AIDS Prevention Research Synthesis (PRS) project at the U.S. Centers for Disease Control and Prevention (CDC) has selected the Project YES! intervention to be added to the Compendium of Evidence-Based Interventions and Best Practices for HIV Prevention for Structural Interventions and Medication Adherence (inclusion forthcoming).

\section{HCP support the Project YES! approach}

HCP support is critical for Project YES! refinement and scale up. Project YES! HCPs across the four participating clinics shared how they valued the YPMs as members of their clinic staff uniquely placed to provide attention and support to AYA that the nurses-in-charge and other clinic staff simply do not have the time or resources to provide.

[The YPMs and I] complemented each other. They were everything I'm not. I'm not an adolescent, they're an adolescent. l'm not going through what they're going through, they're going through it. So, most of the things that I couldn't do for this peer, they were able to.... 


\section{AYA living with HIV experience high levels of violence}

Among HIV-positive AYA in Zambia, an estimated 72 percent of males (95\% Cl 60.3\%-81.2\%) and 75 percent of females (95\% $\mathrm{Cl} 66.6 \%-81.1 \%)$ have experienced some form of physical violence, psychological abuse, or forced sex in the past year. Some, but not all, interviewees qualitatively described how violence influenced their VL (Merrill et al. 2020b). For instance, several experienced psychological abuse due to their HIV status, which spurred thoughts of suicide and hampered medication adherence (Merrill et al. 2021).

\section{YPM as "game changers"}

YPMs discussed how they changed both their clients' lives and their own through Project YES! In terms of their clients, YPM discuss how they shared their struggles and role modeled positive health behaviors: "[It's] not like just talking maybe from a booklet. I'm giving myself as an example...you're helping someone from true life experiences." Additionally, YPMs' experiences resulted in powerful personal transformations in HIV-related health behaviors, conceptions of self, and plans for the future. Many expressed seeing themselves now as community leaders, specifically "ambassadors," "game changers," and "not just alone in this world." YPMs were also adamant that Project YES! should expand so other HIV-positive AYA might benefit, stating that "we just need to continue."

\section{Small grant findings on HIV drug resistance}

\section{The importance of resistance testing}

Project YES! results revealed that in this study population, three out of every four youth with viral failure have at least one HIV drug resistant mutation. This finding has two key implications: a) that potentially a large proportion of youth failing may be resistant to a drug they are currently taking, and b) at least 25 percent of failing youth are failing due to reasons other than resistance, such as incomplete adherence. These factors are critical to take into consideration, particularly with the current emphasis on undetectable=untransmissible and opportunities for differentiated care to provide the support and appropriate medicines for young people with viral failure. Dr. Miti, the lead on this small grant, published these findings as a first-author peer-reviewed publication (Miti et al. 2020). In addition, the findings from Dr. Miti's analysis informed the adoption of new $\mathrm{MOH}$ guidance that HIVDR testing occur at first line failure (instead of the previous guidance to test for resistance at second line failure) as the new standard of care.

\section{RECOMMENDATIONS}

Several recommendations come out of this research.

1. Project YES! YPMS should be incorporated into clinics as a feasible and effective way to increase viral suppression and healthy development among AYA living with HIV. As part of this recommendation, implementation science methods should inform the refinement and scaleup of the Project YES! peer mentoring approach.

2. HIV clinics need to address violence-related needs of AYA attending HIV care. Program options include screening for violence and referring to services, or hiring in-house experts to address 
the impact violence has on the health and HIV outcomes among this particularly vulnerable population.

3. Youth engagement is critical to address the HIV epidemic in sub-Saharan Africa and to successfully achieve UNAIDS 95-95-95 targets. Project YES! offers a concrete evidencedbased approach for engaging youth both as the implementers and as the recipients of Project YES!.

4. The impact of Project YES! in adult clinic settings and the intersection of internalized stigma and viral suppression over time need further exploration. Various biases, including the large percentages lost to follow-up in the adult settings, combined with high viral suppression rates make the findings from the adult settings inconclusive. Future iterations of Project YES! should focus on AYA with viral failure and in tracing lost clients to address these gaps.

5. Project YES! offers a concrete evidence-based approach that engages youth both as implementers and as recipients. Project YES! was selected by the CDC's HIV/AIDS Prevention Research Synthesis Project to be included in the Compendium of Evidence-Based interventions and Best Practices for structural interventions and medication adherence, (inclusion in the Compendium website is forthcoming).

6. HIVDR testing: Project YES! baseline data provided evidence that has since been used to inform changes to the $\mathrm{MOH}$ resistance testing guidance to support HIVDR testing when first line failure occurs. HIVDR data can provide critical information for clinical ART decisions and could inform differentiated care services for AYA with viral failure who have and do not have HIVDR. Dr. Sam Miti has presented these data in Zambia and internationally, and has published them in a peer review article (Miti et al. 2020). 


\section{REFERENCES}

Alvarado, G. et al. 2017. "A systematic review of positive youth development programs in low-and middle-income countries." Washington, DC: Youth Power Learning, Making Cents International.

Denison, Julie A. et al. 2015. "'The sky is the limit': adhering to antiretroviral therapy and HIV selfmanagement from the perspectives of adolescents living with HIV and their adult caregivers," Journal of the International AIDS Society 18(1) :19358. doi: 10.7448/IAS.18.1.19358.

Denison, Julie A., Audrey Pettifor, Lynne Mofenson, and Deanna Kerrigan. 2016. “Developing an implementation science research agenda to improve the treatment and care outcomes among adolescents living with HIV in sub-Saharan Africa," Project SOAR. Washington, D.C.: Population Council. Available at: https://projsoar.org/resources/developing-an-implementation-science-research-agendato-improve-the-treatment-and-care-outcomes-among-adolescents-living-with-hiv-in-sub-saharan-africa/

Denison, Julie. A. et al. 2018. "Factors related to incomplete adherence to antiretroviral therapy among adolescents attending three HIV clinics in the Copperbelt, Zambia," AIDS and Behavior 22(3) 9961005. doi: 10.1007/s10461-017-1944-x

Denison, Julie. A. et al. 2020. “Project YES! Youth Engaging for Success: A randomized controlled trial assessing the impact of a clinic-based peer mentoring program on viral suppression, adherence and internalized stigma among HIV-positive youth (15-24 years) in Ndola, Zambia," PLoS One 15(4): e0230703. doi: 10.1371/journal.pone.0230703

Duffy, Malia H., Heather Bergmann, and Melissa Sharer. 2014. Toolkit for Transition of Care and Other Services for Adolescents Living with HIV. Arlington, VA: USAID's AIDS Support and Technical Assistance Resources, AIDSTAR-One, Task Order 1. Available at: https://www.thecompassforsbc.org/sbcc-tools/ toolkit-transition-care-and-other-services-adolescents-living-hiv

FHI 360. 2013. "Adolescents living with HIV in Zambia: An examination of HIV care and treatment and family planning." Research Triangle Park and Lusaka, Zambia: FHI 360. Retrieved from https://www. fhi360.org/sites/default/files/media/documents/zambia-adolescents-living-hiv-integration-familyplanning.pdf

FHI 360 et al. 2013. "Positive connections: Leading information and support groups for adolescents living with HIV." Durham, NC: FHI 360. Available at: https://www.fhi360.org/sites/default/files/media/ documents/positive-connections-2013.pdf

Government of Zambia. 2013. The Health Services Research Act 2013. Retrieved from http://www. parliament.gov.zm/sites/default/files/documents/acts/Health\%20\%20Research\%20\%20Act\%20 2013.pdf

ISPCAN. 2006. ISPCAN Child Abuse Screening Tool-Child Version. Manual and Proposed Guidelines for Pilot Administration. Aurora, CO: ISPCAN. Retrieved from: https://www.ispcan.org/learn/icastabuse-screening-tools/

Kalichman, S. C. et al. 2009. "Measuring AIDS stigmas in people living with HIV/AIDS: the Internalized AIDS-Related Stigma Scale," AIDS Care 21(1): 87-93. doi: 10.1080/09540120802032627

Lerner, Richard M. et al. 2009. "Positive youth development." In R. J. DiClemente, J. Santelli, and R. A. Crosby (Eds.), Adolescent Health: Understanding and Preventing Risk Behaviors. San Francisco, CA: John Wiley \& Sons.

Lerner, Richard M. and Jacqueline V. Lerner. 2006. "Towards a new vision and vocabulary about adolescence: Theoretical, empirical, and applied bases of a 'Positive Youth Development' perspective." In L. Balter \& C. S. Tamis-LeMonda (Eds.), Child Psychology: A handbook of contemporary issues. Philadelphia: Psychology Press. 
McCarraher, D. R. et al. 2018. "Adolescents living with HIV in the Copperbelt Province of Zambia: Their reproductive health needs and experiences," PLoS One 13(6): e0197853. doi: 10.1371/journal. pone.0197853

Merrill, K. G. et al. 2020a. "Prevalence of physical and sexual violence and psychological abuse among adolescents and young adults living with HIV in Zambia." PLoS One 15 (6): : e0235203. doi: 10.1371/ journal.pone.0235203

Merrill, K. G. et al. 2020b. "Past-year violence victimization is associated with viral load failure among HIV-positive adolescents and young adults," AIDS and Behavior, online ahead of print. doi: 10.1007/ s10461-020-02958-3

Merrill, K. G. et al. 2021. “'So hurt and broken': A qualitative study of experiences of violence and HIV outcomes among Zambian youth living with HIV," Global Public Health, online ahead of print 11 January. doi: 10.1080/17441692.2020.1864749

Ministry of Health. 2019. Zambia Population-based HIV Impact Assessment (ZAMPHIA) 2016: Final Report. Lusaka: Ministry of Health.

Miti, S. et al. 2020. "Prevalence and characteristics of HIV drug resistance among antiretroviral treatment (ART) experienced adolescents and young adults living with HIV in Ndola, Zambia," PLoS One 15(8): e0236156. doi: 10.1371/journal.pone.0236156

Pequegnat, W. et al. 2001. "Measurement of the role of families in prevention and adaptation to HIV/ AIDS," AIDS and Behavior 5(1): 1-19. doi: 10.1023/A:1009557103659

Pequegnat, W. and C. Bell. 2011. Family and HIV/AIDS: Cultural and contextual issues in prevention and treatment. New York: Springer-Verlag New York.

Pequegnat, W. and J. Szapocznik. 2000. Working with Families in the Era of HIV/AIDS. Thousand Oaks, CA: Sage.

Perrino, T., A. González-Soldevilla, H. Pantin, and J. Szapocznik. 2000. "The role of families in adolescent HIV prevention: A review," Clinical Child and Family Psychology Review 3(2): 81-96. doi: 10.1023/A:1009571518900

Thurman, T. R., B. Jarabi, and J. Rice. 2012. "Caring for the caregiver: evaluation of support groups for guardians of orphans and vulnerable children in Kenya," AIDS Care 24(7): 811-819. doi: 10.1080/09540121.2011.644229

UNAIDS. 2014. "Fast-track: ending the AIDS epidemic by 2030." Geneva: UNAIDS. Retrieved from https://www.unaids.org/sites/default/files/media_asset/JC2686_WAD2014report_en.pdf

World Health Organization. 2005. “WHO multi-country study on women's health and domestic violence against women: summary report of initial results on prevalence, health outcomes and women's responses." Geneva: WHO. Retrieved from: https://www.who.int/reproductivehealth/publications/ violence/24159358X/en/

World Health Organization. 2014. "Health for the world's adolescents: A second chance in the second decade." Geneva: WHO. Retrieved from http://apps.who.int/adolescent/second-decade/section2/ page5/adolescence-psychological-and-social-changes.html 
Project SOAR

Population Council 4301 Connecticut Ave, NW,

Suite 280

Washington, D.C. 20008 USA

Tel: +1 2022379400

Fax: +1 2022378410

Ideas. Evidence. Impact.

projsoar.org 